\title{
Silencing of HIV RNA by a Hairpin-loop DNA
} K Moelling ${ }^{* \neq}$, S Abels, J Jendis, J Heinrich, A Ziogas and A Matskevich

\author{
Address: Institute of Medical Virology, University of Zurich, Switzerland \\ Email: K Moelling* - moelling@immv.unizh.ch \\ * Corresponding author ‡Presenting author
}

from 2005 International Meeting of The Institute of Human Virology

Baltimore, USA, 29 August - 2 September 2005

Published: 8 December 2005

Retrovirology 2005, 2(SuppI I):SI54 doi:10.II86/1742-4690-2-SI-SI54

We describe an RNA silencing, which inhibits HIV replication by a hairpin-loop DNA. A partially double-stranded 54mer DNA oligonucleotide (ODN) was targeted to the polypurine tract, PPT of HIV. It inhibits virus replication. We demonstrate that it prevents steps before DNA provirus formation. The effect of the ODN on HIV replication in cell culture is highly sequence-specific and sensitive to changes in length and single mismatches on either strand of the DNA. An ODN against HIV-IIIB was ineffective against HIV-Ba-L and vice versa, whereby their PPT's differ by two of 24 nucleotides. Thus, the structure and sequence of both strands of the ODN are important in cellular assays. In vitro the ODN leads to an RNA-DNA hybrid formation at the PPT, a structure which is cleaved by the RT/ RNase $\mathrm{H}$ in permeabilized virus particles. The hybrid at the PPT is preferentially recognized by the RT/RNase $\mathrm{H}$ for initiation of the second-strand DNA synthesis. This recognition is its normal biological function and shown here with the ODN. A cell extract containing cellular RNase H activities or RISC proteins, is unable to induce such a cleavage. In summary, the ODN mimicks a real step in viral replication, whereby the viral RNA is cleaved prematurely before DNA transcription is completed. The mechanism is reminiscent of RNA silencing by siRNA and supported by its relationship with RNaseH. The ODN may be a basis for drug design, because of low tendency for escape mutations. 